Journal for ImmunoTherapy of Cancer

\title{
Cystathionine $\beta$-synthase mediated PRRX2/IL-6/STAT3 inactivation suppresses Tregs infiltration and induces apoptosis to inhibit HCC carcinogenesis
}

To cite: Zhou Y-F, Song S-S, Tian M-X, et al. Cystathionine $\beta$-synthase mediated PRRX2/ IL-6/STAT3 inactivation suppresses Tregs infiltration and induces apoptosis to inhibit HCC carcinogenesis. Journal for ImmunoTherapy of Cancer 2021;9:e003031. doi:10.1136/ jitc-2021-003031

- Additional supplemental material is published online only. To view, please visit the journal online (http://dx.doi.org/10. 1136/jitc-2021-003031).

Y-FZ, S-SS, M-XT and ZT contributed equally.

Accepted 08 July 2021

Check for updates

(c) Author(s) (or their employer(s)) 2021. Re-use permitted under CC BY-NC. No commercial re-use. See rights and permissions. Published by BMJ.

For numbered affiliations see end of article.

\section{Correspondence to}

Professor Ying-Hong Shi; shi.yinghong@zs-hospital.sh.cn

Dr Wei-Ren Liu;

liu.weiren@zs-hospital.sh.cn

\section{ABSTRACT}

Background Hepatocellular carcinoma (HCC) is characterized by inflammation and immunopathogenesis. Accumulating evidence has shown that the cystathionine $\beta$-synthase/hydrogen sulfide (CBS/ $\left.\mathrm{H}_{2} \mathrm{~S}\right)$ axis is involved in the regulation of inflammation. However, roles of CBS in HCC development and immune evasion have not been systematically investigated, and their underlying mechanisms remain elusive. Here, we investigated the roles of CBS in tumor cells and tumor microenvironment of HCC.

Methods $236 \mathrm{HCC}$ samples were collected to detect the expression of CBS, cleaved Caspase-3 and paired related homeobox 2 (PRRX2) and the number of immune cells. HCC cell lines were employed to examine the effects of CBS on cellular viability, apoptosis and signaling in vitro. Cbs heterozygous knockout mice, C57BL/6 mice, nude mice and non-obese diabetic severe combined immunodeficiency mice were used to investigate the in vivo functions of CBS.

Results Downregulation of CBS was observed in HCC, and low expression of CBS predicted poor prognosis in HCC patients. CBS overexpression dramatically promoted cellular apoptosis in vitro and inhibited tumor growth in vivo. Activation of the $\mathrm{Cbs} / \mathrm{H}_{2} \mathrm{~S}$ axis also reduced the abundance of tumor-infiltrating Tregs, while Cbs deficiency promoted Tregs-mediated immune evasion and boosted tumor growth in Cbs heterozygous knockout mice. Mechanistically, CBS facilitated the expression cleaved Caspase-3 in tumor cells, and on the other hand, suppressed Foxp3 expression in Tregs via inactivating IL-6/STAT3 pathway. As a transcription factor of IL-6, PRRX2 was reduced by CBS. Additionally, miR-24-3p was proven to be an upstream suppressor of CBS in HCC. Conclusions Our results indicate the antitumor function of CBS in HCC by inactivation of the PRRX2/IL-6/STAT3 pathway, which may serve as a potential target for HCC clinical immunotherapy.

\section{BACKGROUND}

Hepatocellular carcinoma (HCC) is the sixth most common cancer and the third leading cause of cancer-related death worldwide in 2020, with approximately 906000 new cases and 830000 deaths. ${ }^{1}$ The development of $90 \%$ of HCCs is related to underlying chronic liver inflammation, which is considered to induce fibrosis and/or subsequent cirrhosis. ${ }^{2}$ Chronic necroinflammation, along with constant cell death, liver regeneration, ${ }^{3}$ and altered immune response, ${ }^{4}$ was responsible for liver fibrosis and tumorigenesis. ${ }^{5}$ Therapeutic challenges of HCC are most frequently chemotherapy resistant and diagnosed at advanced stages. ${ }^{5}{ }^{6}$ In addition, emerging immunotherapies were not so effective in treating HCC. ${ }^{6}$ Therefore, it is of great significance to further investigate the mechanism of HCC development to unearth novel potential targets for the clinical treatment of HCC.

The tumor microenvironment (TME) plays a critical role in HCC initiation and progression. ${ }^{7}$ Three major immunosuppressive cell populations including regulatory $\mathrm{T}$ cells (Tregs), tumor-associated macrophages (TAMs) and myeloid-derived suppressor cells (MDSCs) within TME have been shown to be closely associated with tumorigenesis and evolvement. $^{8-11}$ Tregs, as a dominant inhibitory component in immune system, are able to be co-opted by tumor cells and are prone to repress $\mathrm{T}$ cell activation and proliferation partly through secreting interleukin-10 (IL-10) and highly expressing IL2R/CD25. ${ }^{12}$ TAMs could bind to certain coinhibitory receptors on T cells, such as cytotoxic T-lymphocyte associated protein 4 (CTLA-4). On the other hand, TAMs might facilitate Tregs differentiation to further help tumor cells escaping immunological surveillance. ${ }^{13}$ 
Moreover, the inhibitory effect of MDSCs on $\mathrm{T}$ cell response commonly occurs through upregulation of inducible nitric oxide synthase (iNOS) and promotion of arginase-1 enzyme activity. ${ }^{14}$ In other words, relieving immunosuppression is essential for achieving therapeutic improvements in HCC immunotherapy.

Braunstein $e t a l^{15}$ first isolated cystathionine $\beta$-synthase (CBS) from chicken liver in 1969. CBS was later identified as one of the key enzymes for hydrogen sulfide $\left(\mathrm{H}_{2} \mathrm{~S}\right)$ production, in addition to cystathionine gammalyase (CSE) and 3-mercaptopyruvate sulfurtransferase (3-MST). ${ }^{16}$ Our previous studies have demonstrated that Cbs deficiency-mediated iron overload could lead to liver damage. Meanwhile, we found that interleukin-6 (IL-6) levels in the liver and blood of homozygous knockout mice $\left(\mathrm{Cbs}^{-/}\right)$are higher than that in wild type mice $\left(\mathrm{Cbs}^{+/+}\right)$, and LPS-induced IL-6 production can be inhibited by $\mathrm{H}_{2} \mathrm{~S} .{ }^{17}{ }^{18}$ IL-6, which is known to activate signal transducer and activator of transcription 3 (STAT3), plays a facilitative role in various types of tumors including HCC. ${ }^{19}{ }^{20}$ Nevertheless, the role of CBS in HCC carcinogenesis, especially in TME, remains largely unclear.

Herein, we first explored the effect of CBS on cleaved Caspase-3 and Ki-67 in HCC cells, which are recognized markers for cell apoptosis and proliferation, respectively. ${ }^{21-23}$ We then examined the possible remodeling role of CBS in HCC tumor microenvironment and found an obvious restraint in Tregs infiltration via inhibiting PRRX2/IL-6/STAT3 pathway. Overall, as a tumor suppressor, CBS harbors dual functions in HCC.

\section{METHODS}

\section{Human samples}

Tumor specimens for tissue microarrays were collected from 236 HCC patients who underwent surgery from April 2005 to September 2008 at Department of Liver Surgery, Zhongshan Hospital of Fudan University. All patients were informed of the aim of the study and gave consent to donate their samples.

\section{Statistical analysis}

Two-tailed t-test was used to compare the differences between two sets, and two-way analysis of variance was used to assess the statistical significance of two-factor interactions with multiple time points. The X-tile software was used to generate the optimum cut-off point for continuous variables, ${ }^{24}$ and HCC patients was divided into CBS high expression group and CBS low expression group according to the optimum cut-off point. Kaplan-Meier survival analysis with log-rank test was performed to evaluate risk factors for overall survival (OS) of HCC patients, then multivariate Cox analysis was used to analyze predictors for the prognosis. Data were analyzed with SPSS software V.19.0 (SPSS Inc). $\mathrm{P}<0.05$ was considered statistically significant.

Other experimental procedures are described in the online supplemental material.
RESULTS

Downregulated CBS expression is associated with poor prognosis, less apoptosis and more tumor-infiltrating Tregs in HCC

To explore the role of CBS in the development of HCC, we first examined CBS expressions in different organs. As shown in online supplemental figure 1, both mRNA and protein levels of CBS were considerably higher in liver than in the other seven organs, which implied that CBS might play an essential role in maintaining liver function and homeostasis. Next, we performed statistical analysis on CBS expression in TCGA-LIHC and GEO (GSE14520) databases. Consistent with a previous study, ${ }^{25}$ we observed that the mRNA levels of CBS in HCC tumor tissues from both databases were remarkably lower than that in peritumor tissues (figure 1A,B, left panel), and the OS of HCC patients with low CBS expression was significantly shorter than those with high CBS expression (figure 1A,B, right panel). We also analyzed CBS expression in fresh HCC samples by western blot and real-time PCR, and results showed that both protein and mRNA levels of CBS were remarkably reduced in HCC tumor tissues compared with matched peritumor tissues (figure 1C-E). As CBS is one of the key enzymes involved in $\mathrm{H}_{2} \mathrm{~S}$ production, we also examined $\mathrm{H}_{2} \mathrm{~S}$ concentration. As expected, $\mathrm{H}_{2} \mathrm{~S}$ contents in HCC tumor tissues were much lower than that in adjacent non-tumor tissues (online supplemental figure 2A), then a tissue microarray was employed to examine CBS expressions in 236 HCC patients with different stages. Representative IHC staining of CBS in tumor and peritumor tissues were shown in figure $1 \mathrm{~F}$. It was observed that CBS was mainly localized in the cell cytoplasm. Although CBS was most abundant in normal tissues, it was downregulated in matched HCC tumor tissues (figure 1G). In order to further investigate the role of CBS in HCC development, HCC patients were stratified into different subgroups according to disease stage, and CBS expression was observed to be lower in patients with advanced stage than those with early stage (figure $1 \mathrm{H}$ ). KaplanMeier analysis revealed that patients with low CBS expression had shorter OS than those with high CBS expression (figure 1I). Furthermore, we found that the expression of CBS was positively correlated with the proportion of cleaved Caspase-3 and negatively correlated with the number of Tregs in HCC clinical samples (figure 1J-K). CBS expression was also slightly correlated with the number of $\mathrm{CD}^{+} \mathrm{T}$ cells and TAMs but not with other seven kinds of immune cells including pan T cells, $\mathrm{CD} 8^{+}$ $\mathrm{T}$ cells, mononuclear cells, B cells, plasma cells, naive $\mathrm{T}$ cells and memory $\mathrm{T}$ cells (online supplemental figure 3). These findings indicated that downregulation of CBS might reduce tumor cell apoptosis and increase tumor infiltrating of Tregs in HCC.

Univariate and multivariate analyses were subsequently performed to determine the prognostic factors for the OS of HCC patients. As shown in online supplemental table 5, the levels of alpha fetoprotein, $\gamma$-glutamyl transpeptidase (GGT) and alanine aminotransferase (ALT), 
A

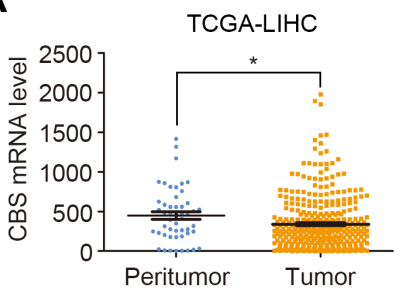

B
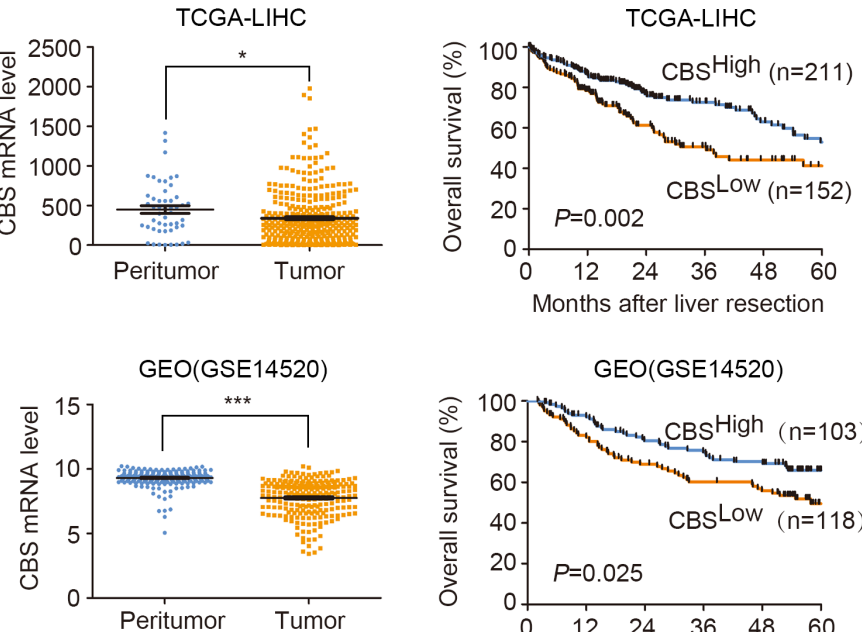

F

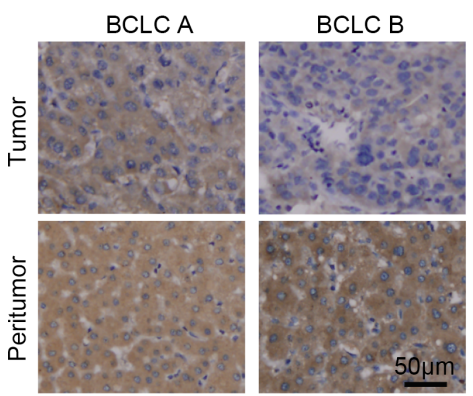

C

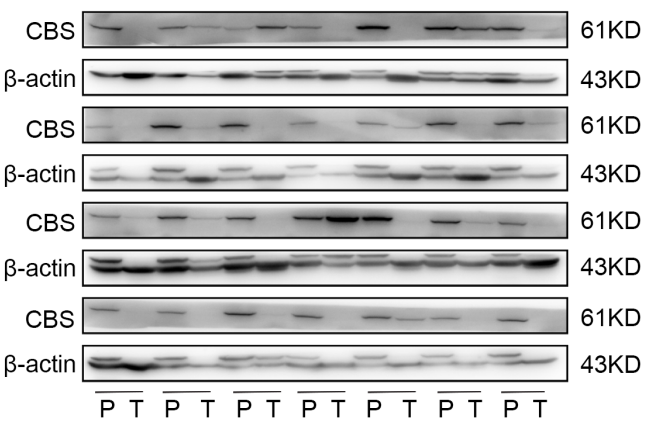

D

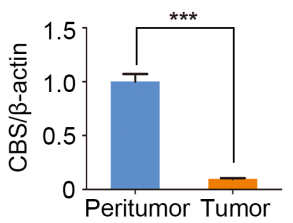

E

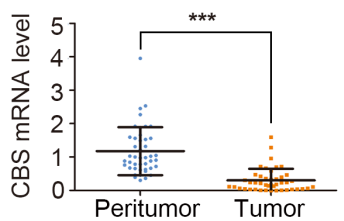

H

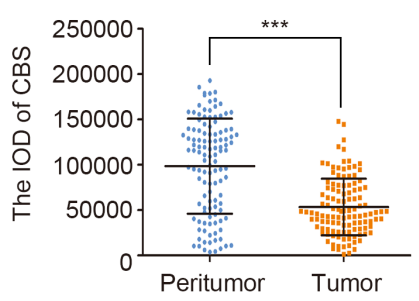

I

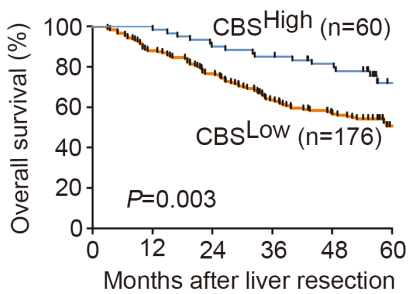

$\mathbf{J}$

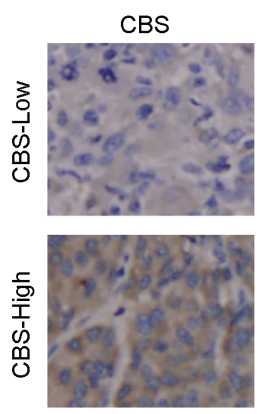

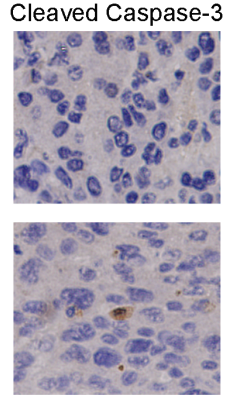

Foxp3

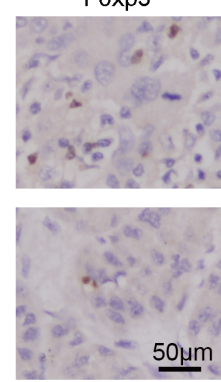

K

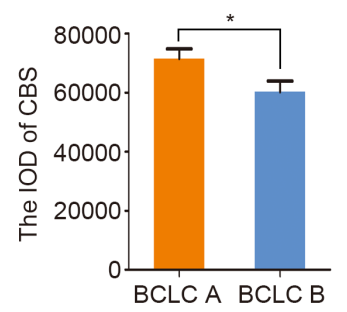

Figure 1 Downregulated CBS expression was associated with poor prognosis, less apoptosis and more tumor-infiltrating Tregs in HCC. (A and B) Relative expression of CBS in HCC tumor versus peritumor tissues and the relationship between CBS expression and OS of HCC patients in TCGA (A) and GSE14520 (B) database, respectively. (C and D) The protein levels of CBS in $28 \mathrm{HCC}$ tumor tissues and paired peritumor tissues were determined by western blot analysis. (E) The mRNA levels of CBS in $44 \mathrm{HCC}$ tumor tissues and paired peritumor tissues were determined by real-time PCR analysis. (F) Representative IHC staining of CBS in HCC tumor tissue and matched non-tumor tissue. (G) The staining score of CBS in tumor tissues compared with peritumor tissues in all patients $(n=118)$. $(H)$ The staining score of CBS in tumor tissues from patients with early stage compared with those with late stage. (I) The relationship between CBS expression and the OS of HCC patients in Zhongshan cohort was determined by Kaplan-Meier analysis with log-rank test $(n=236)$. ( $($ and K) The correlation between CBS expression and the proportion of cleaved caspase-3 as well as the number of Tregs in HCC clinical samples $(n=236)$ was analyzed by Pearson's $r$ test. In A, B, D, E, G and H, data are represented as mean \pm SEM, and statistical significance was calculated by Student's twotailed t-test. ${ }^{\star} \mathrm{P}<0.05 ;{ }^{* \star *} \mathrm{p}<0.001$. CBS, cystathionine $\beta$-synthase; HCC, hepatocellular carcinoma; OS, overall survival.

tumor diameter, tumor differentiation, and CBS expression were identified as risk factors for the OS of HCC patients. Further estimation by multivariate Cox regression analysis demonstrated that GGT level, tumor diameter, tumor differentiation and CBS expression were independent predictors for the OS of HCC patients (online supplemental table 5). Taken together, these results implied that CBS could be a predictor for the OS of patients with HCC.

\section{CBS inhibits the growth of HCC cells in vitro and in vivo}

To investigate the effect of CBS on HCC progression, we transduced lentivirus to achieve stable knockdown of CBS 
in Hep3B cells and overexpression of CBS in MHCC97H cells, and we also transfected Cbs-plasmid into mouse Hepa1-6 cells. Real-time PCR and western blot were performed to confirm the efficiency of CBS knockdown and overexpression (figure 2A-C). CCK-8 assays showed that CBS knockdown promoted the viability of HCC cells, and flow cytometry analysis showed that CBS knockdown inhibited cellular apoptosis (figure 2D,G,H). In contrast, CBS overexpression suppressed the viability and facilitated the apoptosis of HCC cells (figure 2E,F,I-L). Subcutaneous xenograft tumor model was used to verify the antitumor role of CBS in HCC. Results showed that CBS knockdown or inhibition by aminooxyacetic acid (AOAA; a CBS inhibitor $)^{26}$ significantly enhanced tumor growth in Hep3B-xenografted mice model, while CBS overexpression or activation by S-adenosyl methionine (SAM; a CBS agonist) ${ }^{27}$ exhibited opposite effects in MHCC97Hxenografted mice model (figure 3A,B,E,F). Moreover, $\mathrm{H}_{2} \mathrm{~S}$ contents in subcutaneous tumor tissues were declined following CBS depletion and were increased following CBS activation (online supplemental figure 2B,C).

We further detected the influence of CBS on the cell apoptosis marker cleaved Caspase-3 and the cell proliferation marker Ki-67 in these subcutaneous HCC tumor tissues. As shown in figure 3C,G, CBS depletion remarkably diminished cleaved Caspase-3 positive rate, while CBS activation improved the proportion of cleaved Caspase-3. Nevertheless, CBS depletion or activation showed little effect on the expression of Ki-67 (figure 3D,H). A previous study reported the regulation of CBS in glycolysis or angiogenesis, ${ }^{28}$ which play crucial roles in tumor progression. Extracellular acidification rate calculated from proton production rate was used to evaluate glycolytic capacity, ${ }^{29} 30$ and CD31 staining was used to identify tumor angiogenesis. ${ }^{31}$ However, unlike reported results in colorectal and ovarian cancers, ${ }^{28}$ our results showed that CBS knockdown or overexpression exhibited little effect on glycolysis or angiogenesis in HCC (online supplemental figure 4). Together, these data suggested that CBS suppressed HCC tumor growth through inducing cellular apoptosis but had no effects on cell proliferation, glycolysis or angiogenesis.

\section{CBS suppresses tumor growth by reducing tumorous infiltration of Tregs}

Next we used the CBS agonist SAM and a slow $\mathrm{H}_{2} \mathrm{~S}$ donor GYY4137 to further verify the effects of $\mathrm{CBS} / \mathrm{H}_{2} \mathrm{~S}$ axis activation on Hepa1-6-homografted tumor inhibition in different mice strains. ${ }^{27}{ }^{32}$ As shown in figure $4 \mathrm{~A}$, both SAM and GYY4137 administration impaired tumor growth in immunocompetent C57BL/6 mice as well as in non-obese diabetic severe combined immunodeficiency (NOD SCID) mice. Interestingly, tumor inhibition rates under GYY4137 or SAM treatment were significantly higher in immunocompetent mice than that in immunodeficient mice, which might be due to a stronger apoptosis-induced capacity in immunocompetent mice (figure 4B-D). These data suggested a possible immunoregulatory role of the $\mathrm{CBS} / \mathrm{H}_{2} \mathrm{~S}$ axis in tumor growth repression. Considering that CBS expression was negatively correlated with tumor-infiltrating Tregs and TAMs in HCC patients (figure $1 \mathrm{~K}$ and online supplemental figure 3B), we next examined the influence of $\mathrm{CBS} / \mathrm{H}_{2} \mathrm{~S}$ activation on the populations of Tregs and TAMs as well as another immunosuppressive MDSCs in tumor microenvironment. Flow cytometry analysis indicated that the abundance of tumor-infiltrating Tregs in subcutaneous tumors derived from immunocompetent mice treated with GYY4137 or SAM was significantly reduced compared with control mice (figure $4 \mathrm{E}$ and online supplemental figure 5A). However, MDSCs and TAMs were almost unchanged after GYY4137 or SAM administration (online supplemental figure 5B,C). To confirm the effect of CBS on the immunosuppressive function of Tregs, we detected the abundance of tumorinfiltrating IFN $\gamma^{+} \mathrm{CD}^{+} \mathrm{T}$ cells, and results showed that both GYY4137 and SAM treatment significantly increased the proportion of IFN $\gamma^{+} \mathrm{CD} 8^{+} \mathrm{T}$ cells (figure $4 \mathrm{~F}$ and online supplemental figure 5D). In GYY4137 and SAM-treated tumor tissues, the inhibition of $\mathrm{CD}^{+} \mathrm{T}$ cell proliferation by Tregs was impaired compared with control group (figure 4G). Furthermore, the tumor killing capacity of $\mathrm{CD}^{+} \mathrm{T}$ cells was higher in GYY4137 and SAM-treated group compared with control group (figure $4 \mathrm{H}$ ). The mRNA levels of IL-10 in Tregs and IFN $\gamma$ in $\mathrm{CD}^{+} \mathrm{T}$ cells were also examined, and results showed that GYY4137 and SAM treatment significantly diminished IL-10 mRNA level in Tregs but accelerated IFN $\gamma$ expression in $\mathrm{CD}^{+} \mathrm{T}$ cells (online supplemental figure $5 \mathrm{E}, \mathrm{F}$ ).

Cbs-deficient $\left(\mathrm{Cbs}^{+/-}\right)$immunocompetent mice were used to further certify the suppressive roles of CBS in HCC. As shown in figure 4I-L, Cbs deficiency notably facilitated Hepa1-6-homografted tumor growth and abnormally elevated the tumorous infiltration of Tregs while reduced tumor-infiltrating IFN $\gamma^{+} \mathrm{CD} 8^{+} \mathrm{T}$ cells. Similar to GYY4137 and SAM treatment, Cbs deficiency exhibited little effect on the abundance of tumor-infiltrating MDSCs and TAMs (online supplemental figure 5G,H). In addition, Tregs separated from $\mathrm{Cbs}^{+/-}$mice showed stronger suppression on the proliferation of $\mathrm{CD}^{+} \mathrm{T}$ cells than those from wild type group (figure $4 \mathrm{M}$ ), and the tumor-killing capacity of $\mathrm{CD}^{+} \mathrm{T}$ cells derived from $\mathrm{Cbs}^{+/-}$mice was weakened compared with those from wild type group (figure $4 \mathrm{~N}$ ). Cbs deficiency enhanced IL-10 expression in Tregs while repressed IFN $\gamma$ expression in $\mathrm{CD}^{+} \mathrm{T}$ cells (online supplemental figure 5I,J). Furthermore, we constructed a primary liver carcinogenesis mouse model with intraperitoneal injection of the chemical carcinogen diethylnitrosamine (DEN). As shown in online supplemental figure $5 \mathrm{~K}$, after a short-induced period (4 months), no tumor lesions were observed in wild type mice, while $33.3 \%$ of the Cbs-deficient mice developed microtumor lesions. These data implied that Cbs deficiency improved DEN-induced HCC incidence. 
A

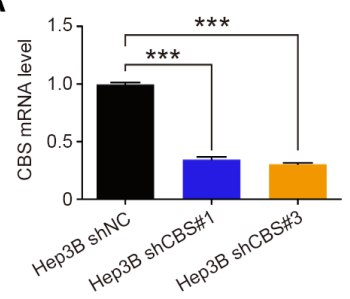

B

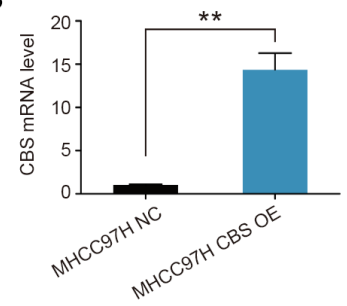

C

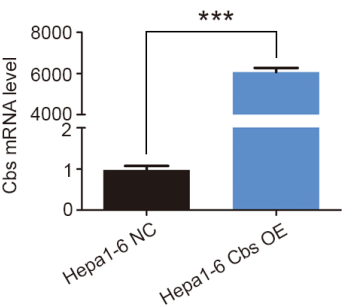

G

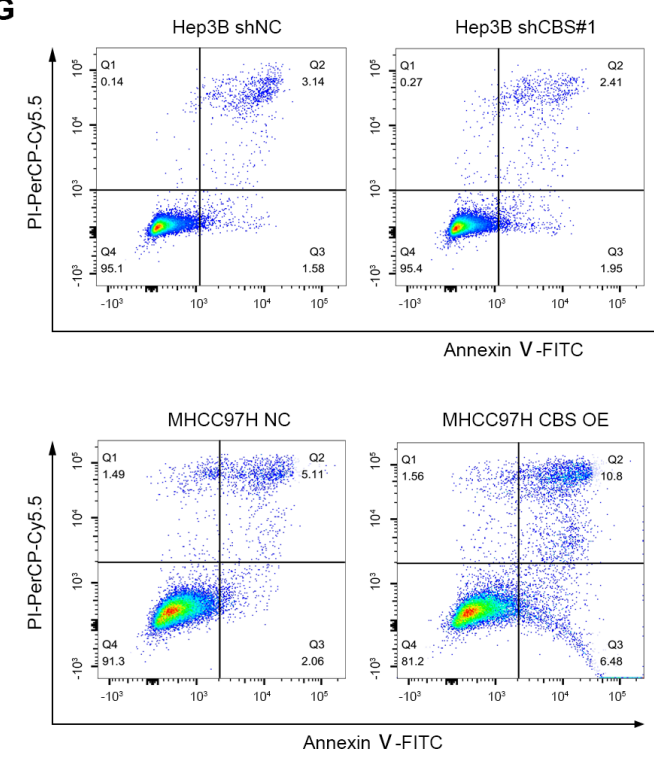

K

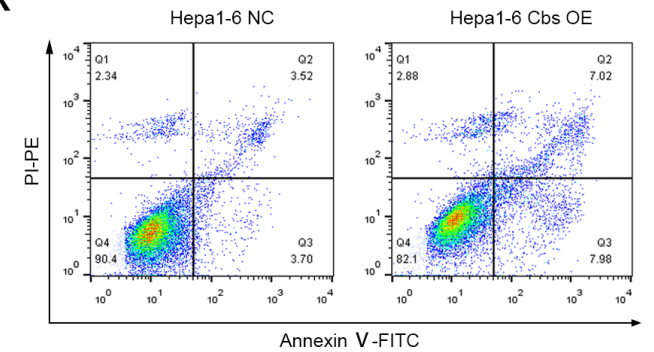

I

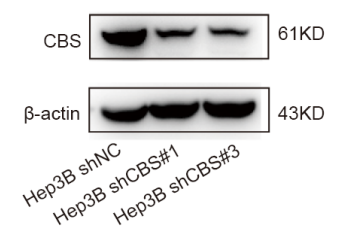

D

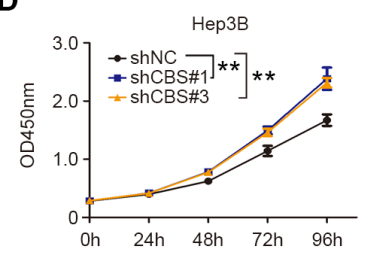

E
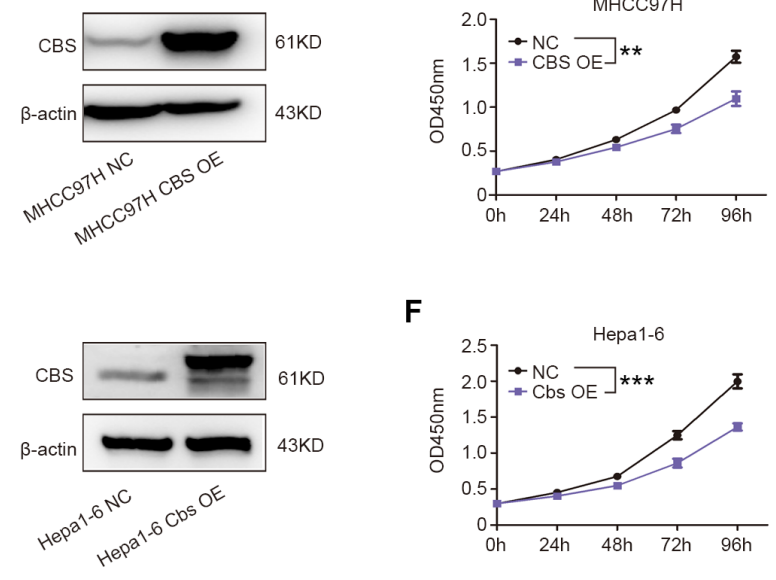

H

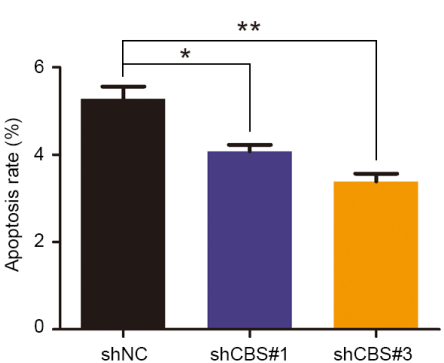

J

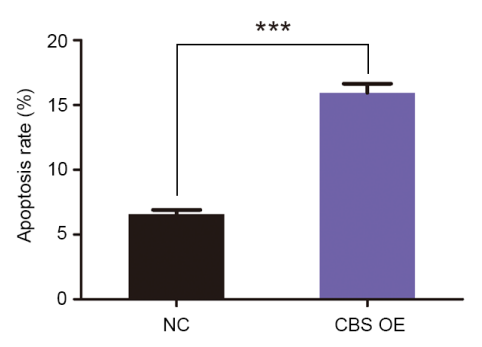

L

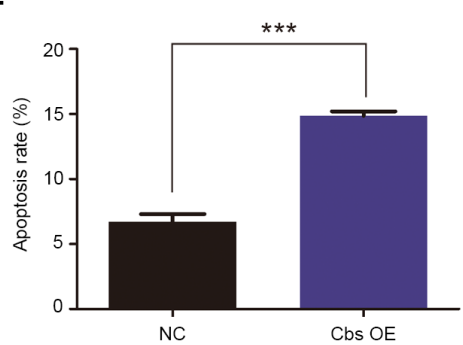

Figure 2 CBS inhibits cell viability and promotes apoptosis of HCC cells. (A-C) The knockdown efficiency of CBS in Hep3B cells (A) and overexpression efficiency of CBS in MHCC97H (B) and Hepa1-6 cells (C), respectively. (D-F) The effect of CBS knockdown on Hep3B cell viability (D) and the effect of CBS overexpression on MHCC97H (E) and Hepa1-6 cell viability $(\mathrm{F})$ were detected by using CCK-8 assay, respectively. (G-L) Flow cytometry was used to determine apoptosis of Hep3B, MHCC97H and Hepa1-6 cells, and representative images were shown in G, I and $\mathrm{K}$. In A-L, data are represented as mean $\pm S E M$, and statistical significance was calculated by Student's two-tailed t-test ( $\mathrm{A}-\mathrm{C}$ and $\mathrm{H}, \mathrm{J}$ and $\mathrm{L}$ ) or two-way ANOVA analysis (D-F). ${ }^{*} \mathrm{P}<0.05$; ${ }^{* \star} p<0.01 ;{ }^{* \star} p<0.001$. ANOVA, analysis of variance; CBS, cystathionine $\beta$-synthase; HCC, hepatocellular carcinoma. 
A

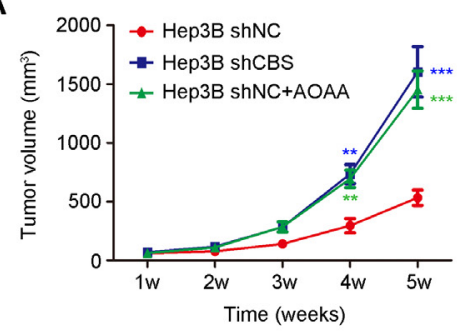

C

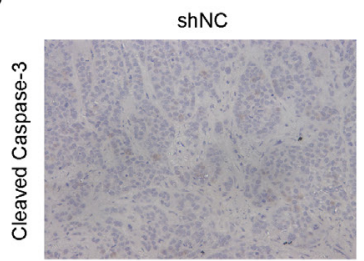

D

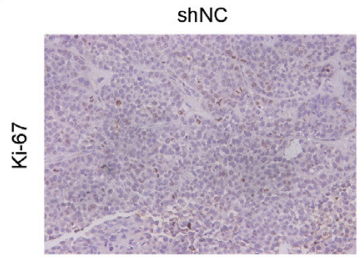

B

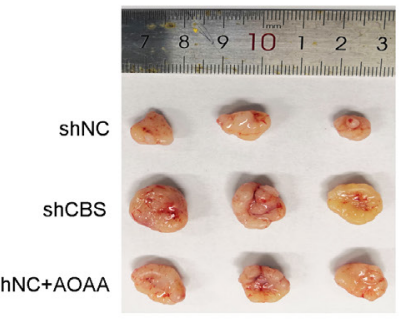

shCBS

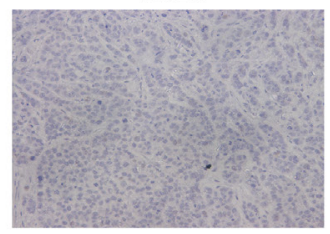

shCBS
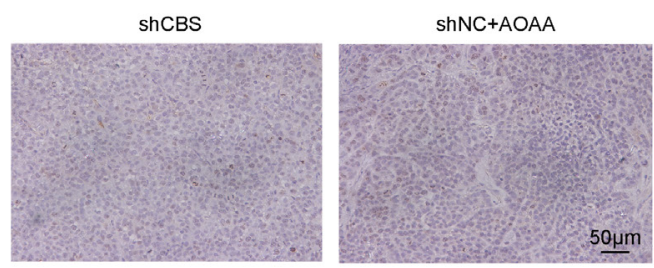
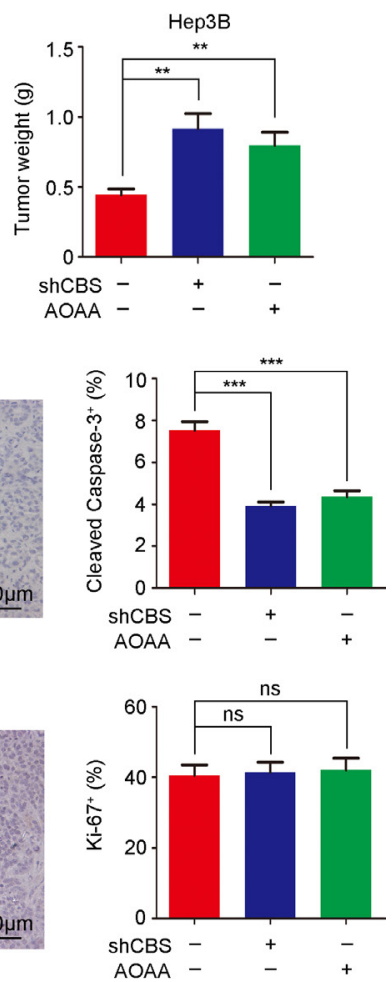

E
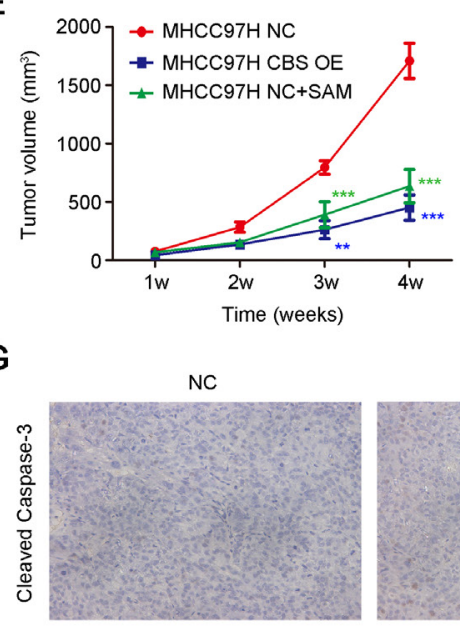

H

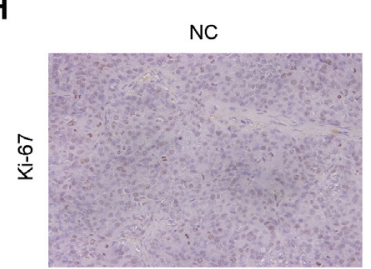

F

NC

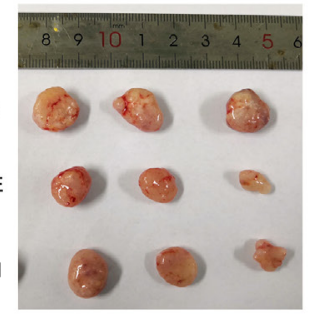

CBS OE

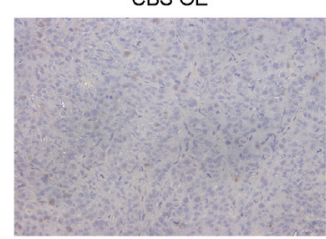

CBS OE

NC+SAM

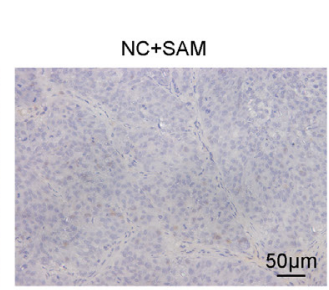

CBS OE

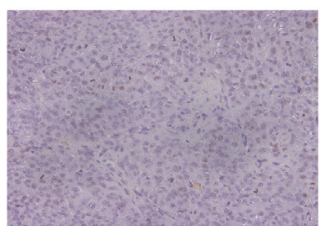

NC+SAM

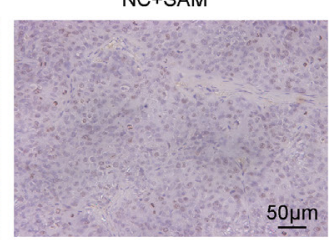

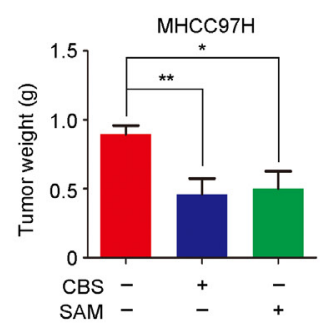
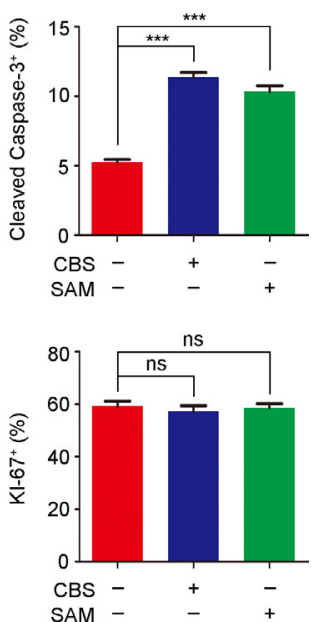

Figure 3 CBS inhibits HCC growth in vivo. (A-D) Nude mice were subcutaneously injected with Hep3B cells with stable CBS knockdown or empty vector and treated with or without AOAA. Tumor volume was measured weekly $(A)(n=6)$. (B) Five weeks later, tumor xenografts were excised and weighed, and representative images are shown (left panel). (C and D) IHC staining was performed to detect cleaved caspase-3 (C) and Ki-67 expression (D). Scale bar: $50 \mu \mathrm{m}$. (E-H) Nude mice were subcutaneously injected with MHCC97H cells with stable CBS expression or empty vector and treated with or without SAM, tumor volume was measured weekly $(E)(n=6)$. (F) Four weeks later, tumor xenografts were excised and weighed, and representative images are shown (left panel). (G and H) IHC staining was performed to detect cleaved Caspase-3 $(G)$ and Ki-67 expression $(H)$. Scale bar: $50 \mu \mathrm{m}$. In $\mathrm{A}-\mathrm{H}$, data are represented as mean $\pm \mathrm{SEM}$, and statistical significance was calculated by Student's two-tailed t-test. ${ }^{*} \mathrm{P}<0.05 ;{ }^{* \star} \mathrm{p}<0.01 ;{ }^{* * *} \mathrm{p}<0.001$. AOAA, aminooxyacetic acid; CBS, cystathionine $\beta$-synthase; HCC, hepatocellular carcinoma; NS, no significance; SAM, S-adenosyl methionine. 


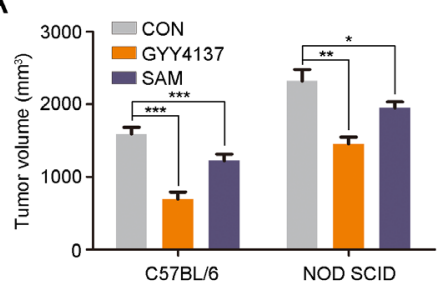

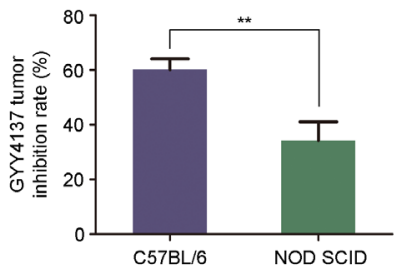

C

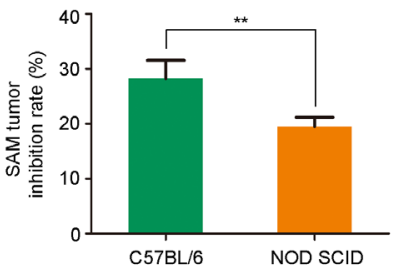

D

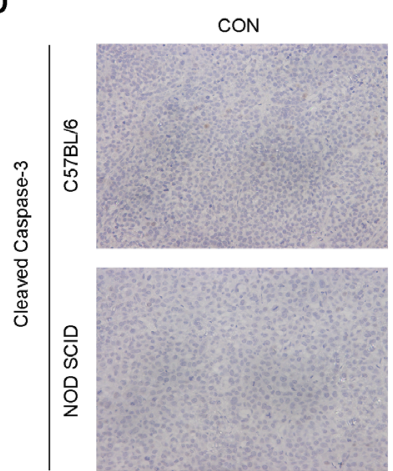

E

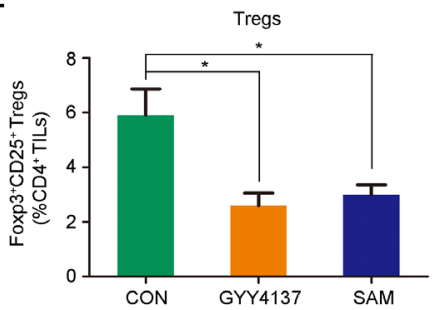

$\mathrm{H}$

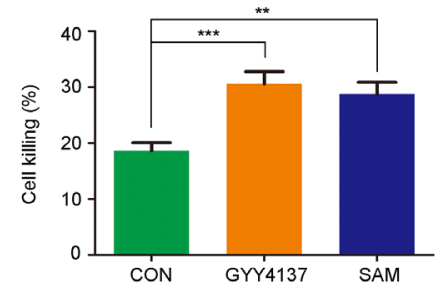

GYY4137

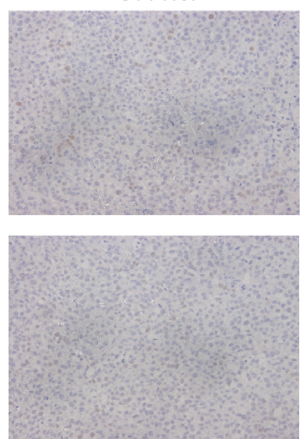

F

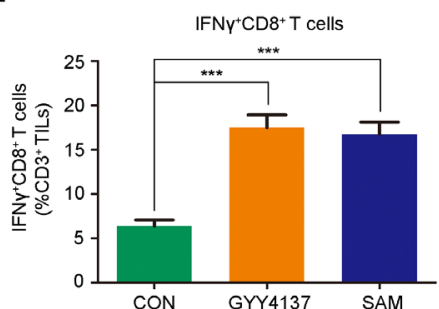

I

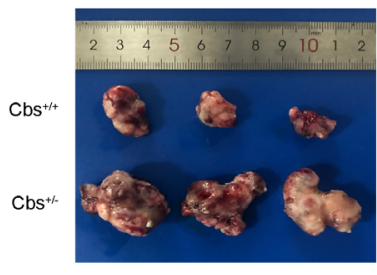

SAM
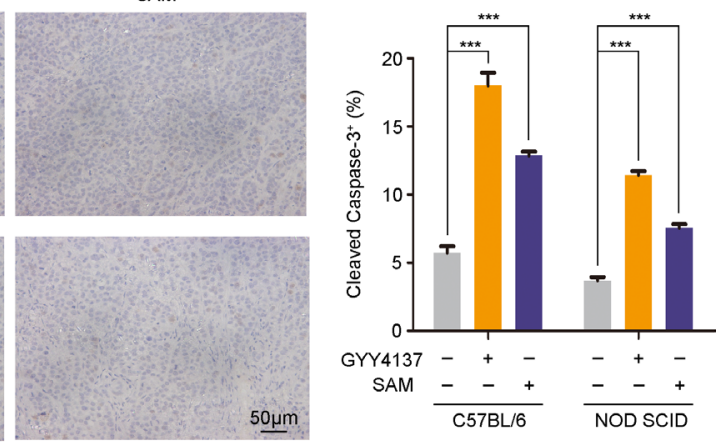

G
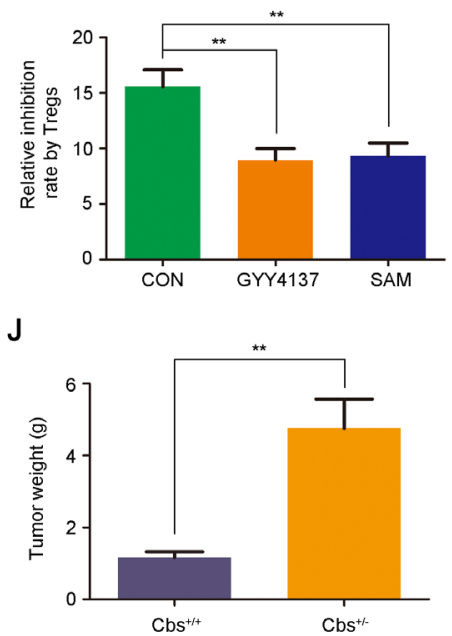

$\mathrm{N}$

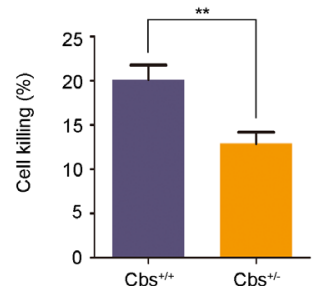

M

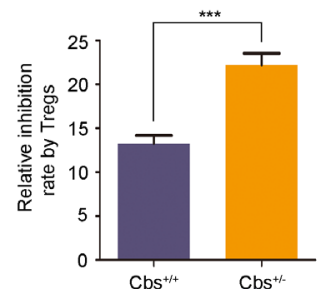

K

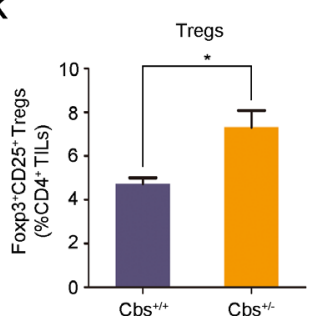

L

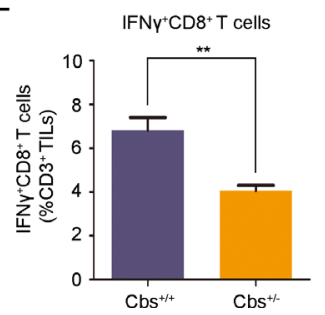

Figure 4 CBS suppresses tumor growth by reducing tumorous infiltration of Tregs. (A-H) Hepa1-6 cells were injected subcutaneously into C57BL/6 and NOD SCID mice treated with or without GYY4137 or SAM for 3 weeks $(n=6)$. (A) Tumor volumes in C57BL/6 and NOD SCID mice treated with or without GYY4137 or SAM. (B) Tumor inhibition rates by GYY4137 in C57BL/6 and NOD SCID mice. (C) Tumor inhibition rates by SAM in C57BL/6 and NOD SCID mice. (D) IHC staining was performed to detect cleaved Caspase-3 expression in subcutaneous tumor tissues. Scale bar: $50 \mu \mathrm{m}$. (E and F) Abundance of regulatory T cells $(\mathrm{E})$ and IFN $\gamma^{+} \mathrm{CD}^{+} \mathrm{T}$ cells $(\mathrm{F})$ in subcutaneous tumor tissues. (G) Tregs inhibition on the proliferation of $\mathrm{CD} 8^{+}$ T cells sorted from the same tumor tissues as indicated. $(\mathrm{H})$ Cell killing assays of $\mathrm{CD}^{+} \mathrm{T}$ on Hepa1-6 cells separated from C57BL/6 mice treated with or without GYY4137 or SAM. (I-N) Hepa1-6 cells were injected subcutaneously into Cbs-deficient C57BL/6 mice and wild type C57BL/6 mice ( $\mathrm{n}=6$ ). (l) Representative images of excised tumor homografts. (J) Tumor weight of excised tumor homografts. ( $K$ and $L$ ) Abundance of regulatory $T$ cells $(K)$ and IFN $\gamma^{+} \mathrm{CD}^{+} \mathrm{T}$ cells $(\mathrm{L})$ in subcutaneous tumor tissues. (M) Tregs inhibition on the proliferation of $\mathrm{CD}^{+}{ }^{+}$cells sorted from the same tumor tissues as indicated. (N) Cell killing assays of $\mathrm{CD} 8^{+} \mathrm{T}$ on Hepa1-6 cells separated from $\mathrm{Cbs}^{+/-}$mice or wild type mice. In A-H and $\mathrm{J}-\mathrm{N}$, data are represented as mean \pm SEM, and statistical significance was calculated by Student's two-tailed t-test. ${ }^{*} P<0.05 ;{ }^{* \star} p<0.01 ;{ }^{* *} p<0.001$. NOD SCID, non-obese diabetic severe combined immunodeficiency; SAM, S-adenosyl methionine. 
CBS inhibits tumor growth and Tregs infiltration via suppressing STAT3 pathway in HCC

To explore the underlying mechanisms of how CBS restrain HCC progression, we conducted RNA sequencing and human phospho-kinase array in MHCC97H-CBS OE cells in comparison with MHCC97H-NC cells to screen out potential CBS-regulated signal pathways. Gene set enrichment analysis based on RNA sequencing demonstrated that CBS overexpression was significantly negatively related to STAT3 signaling pathway (figure 5A). Consistently, the phospho-kinase array also manifested an obviously attenuation of STAT3 (Y705) phosphorylation when CBS was overexpressed (figure 5B). Western blot results confirmed that CBS overexpression inhibited STAT3 phosphorylation, while knockdown of CBS facilitated STAT3 phosphorylation (figure 5C). Meanwhile, the expression of the apoptosis marker cleaved Caspase-3 was significantly reduced, and the antiapoptosis factor Bcl-3 was increased when CBS was knockdown in Hep3B cells, while opposite trends were observed when CBS was overexpressed in MHCC97H and Hepa1-6 cells (figure 5C), then we aimed to confirm whether CBS depletionmediated tumor growth acceleration was STAT3 dependent. As shown in figure 5D, the STAT3 inhibitor Stattic completely reversed the promotion of cell viability induced by CBS knockdown. The inhibition of cellular apoptosis and cleaved Caspase-3 expression as well as the improvement of Bcl-3 expression induced by CBS depletion were also blocked by Stattic (figure 5E,F). Furthermore, intraperitoneal injection of Stattic reversed the facilitation of tumor growth in mice when CBS was knockdown or partly deficient (figure $5 \mathrm{G}, \mathrm{H}$ ). Cbs deficiencymediated tumor infiltration of Tregs was also disrupted by Stattic (figure 5I). Interestingly, we found that the expression of Foxp3 in $\mathrm{CD} 4{ }^{+} \mathrm{CD} 25^{+}$Tregs was increased by Cbs depletion in a STAT3-dependent manner (figure 5J).

Recent studies indicated that the development and function of Tregs are governed by particularly DNA demethylation of Foxp3 promoter, ${ }^{33}$ and ten-eleven translocation (Tet) family is able to convert 5-methylcytosine to 5-hydroxymethylcytosine $(5 \mathrm{hmC})$ to remove existing methylation marks. ${ }^{34} \mathrm{H}_{2} \mathrm{~S}$ was also shown to promote methylcytosine dioxygenases Tet1-mediated and Tet2-mediated Foxp3 demethylation in spleen and lymph nodes-derived $\mathrm{CD}^{+}{ }^{\mathrm{T}}$ cells. ${ }^{35}$ To clarify whether the upregulation of Foxp3 in tumor-infiltrating $\mathrm{CD} 4^{+} \mathrm{CD} 25^{+}$Tregs mediated by Cbs depletion was also related to DNA demethylation, we examined the levels of Tet1, Tet2 and $5 \mathrm{hmC}$ in tumor infiltrating $\mathrm{CD} 4^{+} \mathrm{T}$ cells. However, the expression of all these factors was almost unchanged following Cbs depletion (online supplemental figure 6A,B). Instead, the mRNA level of IL- 6 was increased in Cbs deficient tumor-infiltrating Tregs (online supplemental figure 6C). These results suggested that Cbs deficiency-mediated IL-6/STAT3 activation promoted the expression of Foxp3 in tumor-infiltrating Tregs. Additionally, we investigated the role of STAT3 in the influence of Cbs deficiency on IFN $\gamma^{+} \mathrm{CD}^{+} \mathrm{T}$ cells. As shown in figure $5 \mathrm{~K}$, L, the reduction of tumor-infiltrating $\mathrm{IFN}^{+} \mathrm{CD}^{+} \mathrm{T}$ cells following $\mathrm{Cbs}$ depletion was reversed by Stattic administration, while tumor killing capacity of IFN $\gamma^{+} \mathrm{CD} 8^{+} \mathrm{T}$ cells was remarkably enhanced by STAT3 inhibition.

\section{CBS negatively regulates STAT3 phosphorylation via PRRX2- transcripted IL-6}

Next we aimed to elaborate how CBS represses STAT3 activation. Numerous studies have confirmed the regulatory effect of CBS on IL- $6^{1736}$ and the activation of STAT3 by IL-6. ${ }^{19}$ As shown in online supplemental figure 7, the mRNA level of IL-6 was reduced when CBS was overexpressed in HCC cells and was increased when CBS was knockdown. Real-time PCR and ELISA analysis also showed that CBS knockdown or inhibition significantly stimulated IL-6 production in subcutaneous tumor tissues, while CBS overexpression or activation inhibited IL-6 expression (figure 6A). Further exploration using IL-6 recombinant protein indicated that CBS-mediated STAT3 inhibition was IL-6 dependent (figure 6B). However, the specific mechanism of CBS regulation on IL-6 was unknown. To elucidate the upstream transcription factor of IL-6, we searched the Jaspar database and found that 93 transcription factors had sequences complementary to IL-6 promotor (online supplemental table 6 ). We then conducted a transcription factors array on these 93 factors in CBS overexpression $\mathrm{MHCC97H}$ cells in comparison with control cells, in order to screen out potential transcription factors. The results showed that 14 transcription factors were significantly reduced along with CBS overexpression (figure 6C). Further realtime PCR analysis verified that only Jun proto-oncogene (JUN), JunB proto-oncogene (JUNB), NK2 homeobox 5 (NKX2-5) and PRRX2 were significantly decreased after CBS overexpression (figure 6D). Moreover, among these four transcription factors, only PRRX2 transfection completely reversed the inhibition of IL- 6 by CBS overexpression (figure $6 \mathrm{E}, \mathrm{F}$ ). We also performed a rescue experiment in CBS knockdown Hep3B cells. As shown in figure $6 \mathrm{G}, \mathrm{H}$, CBS knockdown significantly facilitated IL-6 expression, but PRRX2 depletion blocked this effect. When PRRX2 was regained by plasmid transfection, the mRNA level of IL-6 was rescued subsequently. In addition, the expression of PRRX2 exhibited significant negative correlation with the expression of CBS in tumor tissues from 236 HCC patients (figure 6I,J). Taken together, these data implied that CBS inhibited the expression of PRRX2. As a potential transcription factor, PRRX2 modulated IL-6 transcription and activated downstream STAT3 in HCC cells.

\section{miR-24-3p is an upstream suppressor of CBS}

miRNAs have been demonstrated as important upstream regulators of many tumorigenesis-related genes. To clarify why CBS is downregulated in HCC and elucidate the upstream regulators of CBS, we searched six public databases, including TargetScan, miRanda, miRMap, PITA, RNA22 and RNAhybrid. Among all these six databases, 
A

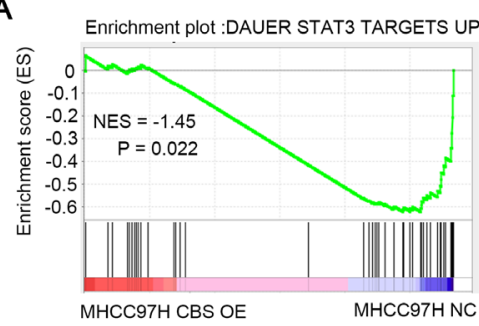

C

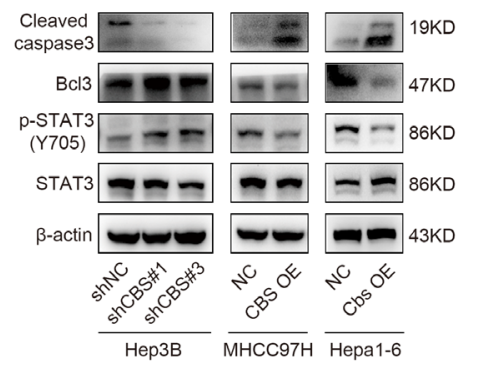

B

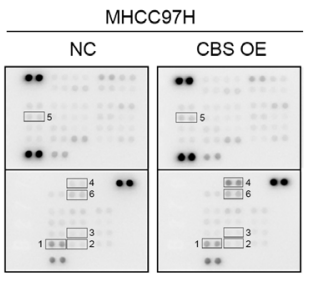

D

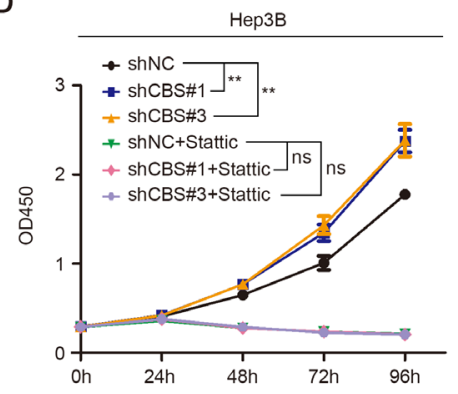

E
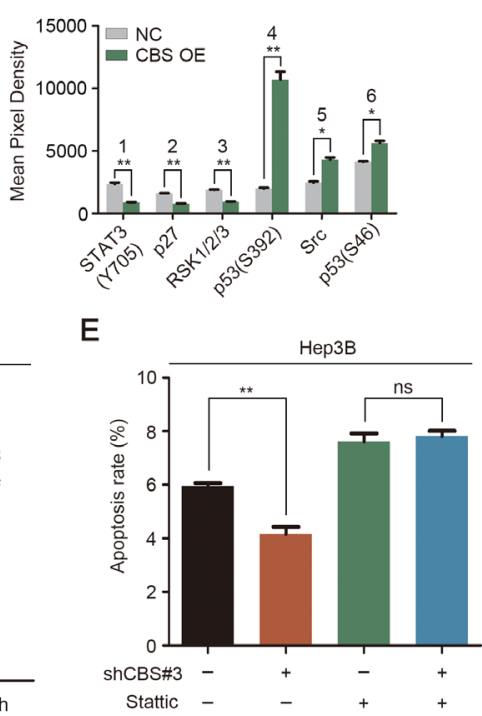

F

Cleaved
caspase3 $3 \cdots \cdots$

BC13 -

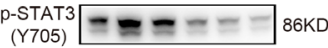

STAT3

СВS $-\cdots \cdots$ 61К

$\beta$-actin $-\infty-\infty-\infty$ 43KD

shCBS\#1 - + - + -

ShCBS\#3 - +--+

Stattic $--\quad+++$

$\mathrm{H}$

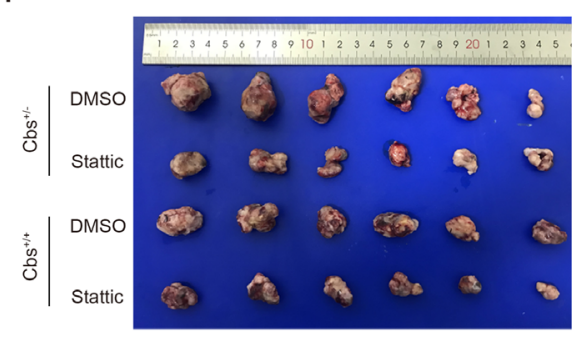

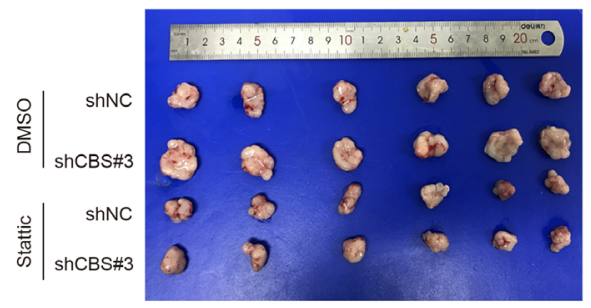

Hep3B

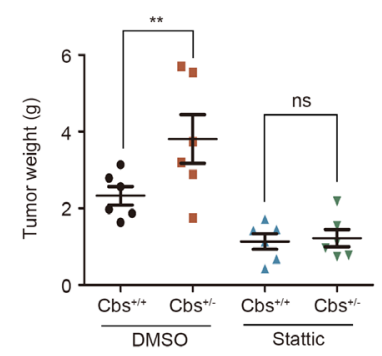

J

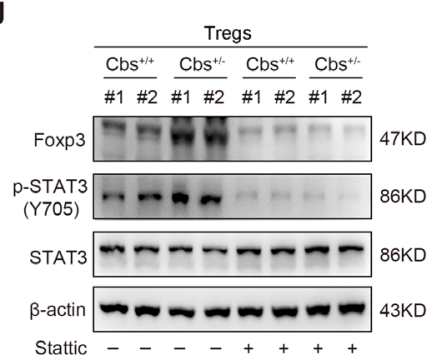

K

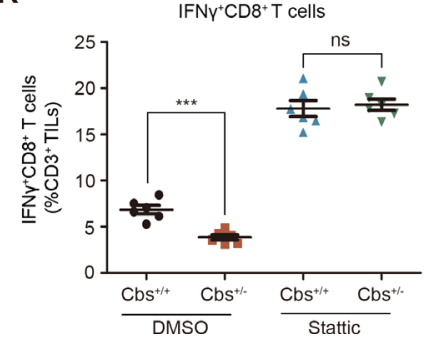

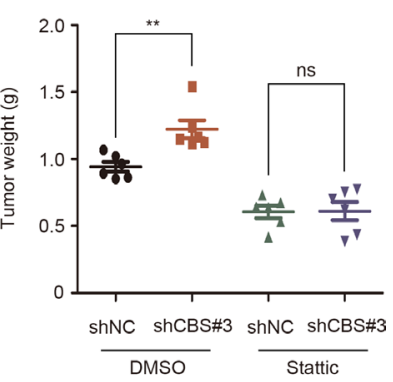

I

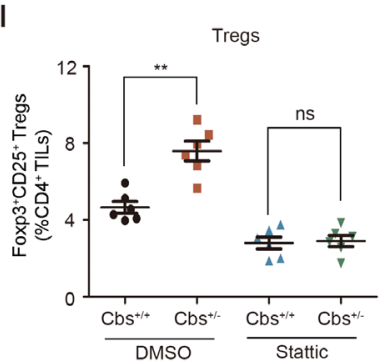

Figure 5 CBS inhibits tumor growth and Tregs infiltration via suppressing STAT3 pathway in HCC. (A) GSEA of the STAT3 downstream pathway (normalized enrichment score (NES)) after CBS overexpression. (B) The phospho-kinase array analysis of MHCC97H cells with stable CBS overexpression or empty vector. (C) The effect of CBS on STAT3 phosphorylation and cleaved Caspase-3 as well as Bcl-3 expression were examined by western blot. (D-F) The effects of Stattic on CBS knockdownmediated cell viability, apoptosis and STAT3 signaling were analyzed by CCK-8 (D), flow cytometry (E) and western blot (F), respectively. ( $G$ and $H$ ) Stattic significantly reversed the growth of subcutaneous tumor accelerated by CBS knockdown (G) or CBS deficiency $(\mathrm{H})$. (I-L) The effect of Stattic on CBS deficiency-mediated tumor infiltration of Tregs (I) and the expression of FOXP3 in Tregs $(\mathrm{J})$ as well as tumor infiltration of IFN $\gamma^{+} \mathrm{CD} 8^{+} \mathrm{T}$ cells $(\mathrm{K})$ and the tumor killing capacity of $\mathrm{CD} 8^{+} \mathrm{T}$ cells $(\mathrm{L})$. In $\mathrm{B}$, $\mathrm{D}, \mathrm{E}, \mathrm{G}-\mathrm{I}$ and $\mathrm{K}-\mathrm{L}$, data are represented as mean $\pm \mathrm{SEM}$, and statistical significance was calculated by two-way ANOVA analysis (D) or Student's two-tailed t-test (B, E, G-I and K-L). ${ }^{\star *} \mathrm{P}<0.01$; ${ }^{* \star} \mathrm{p}<0.001$. ANOVA, analysis of variance; CBS, cystathionine $\beta$-synthase; NS, no significance. 
A
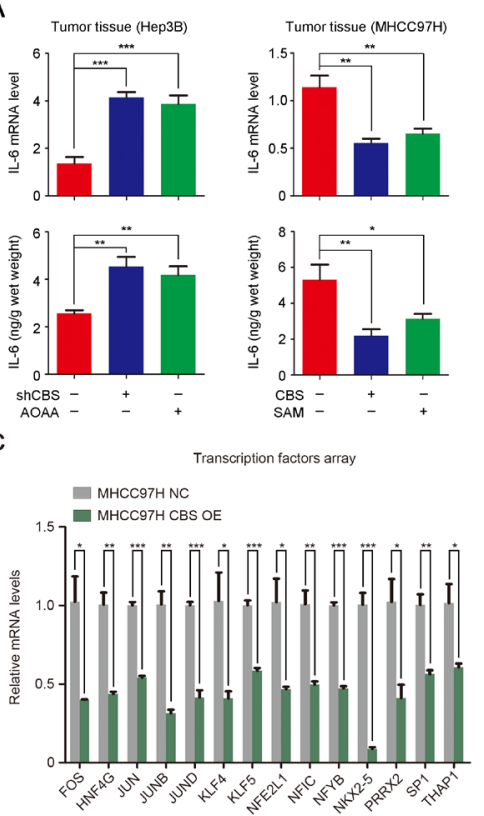

E
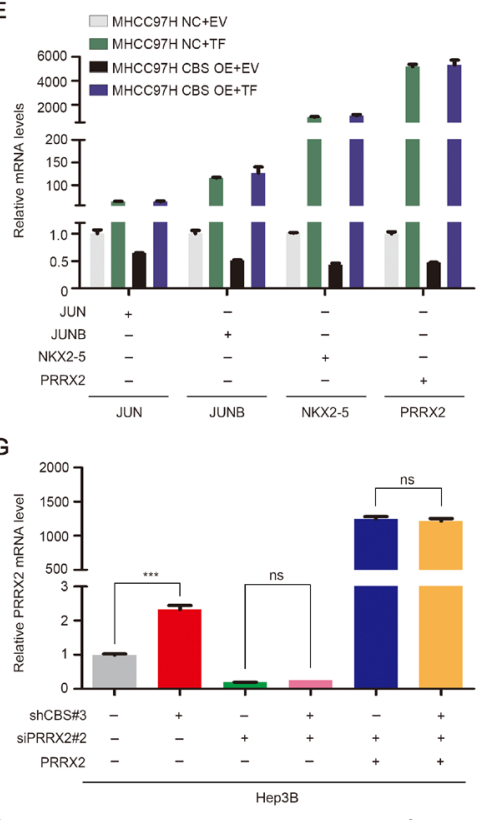
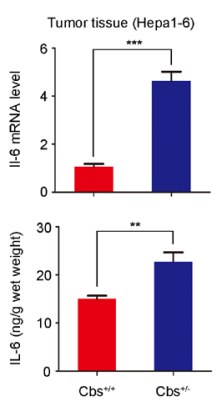

D

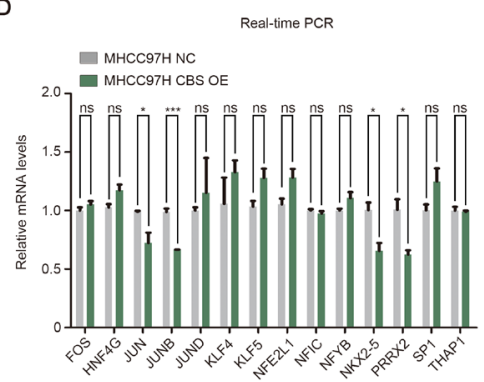

F
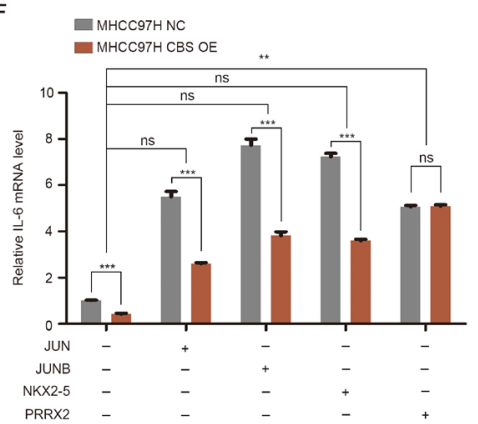

$\mathrm{H}$

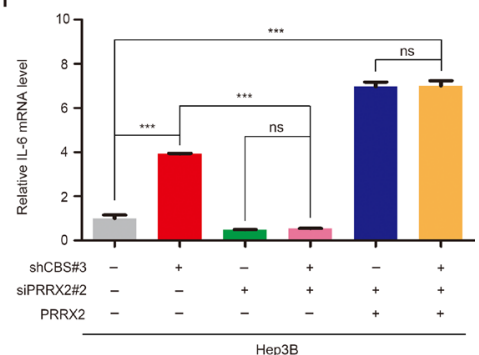

B

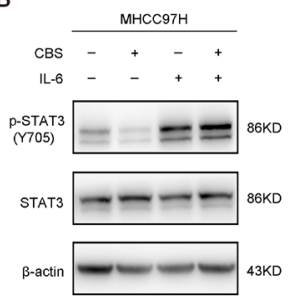

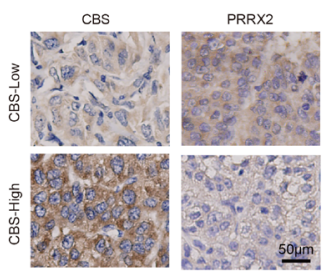

J

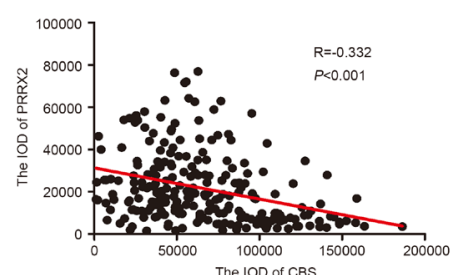

Figure 6 CBS negatively regulates STAT3 phosphorylation via PRRX2-transcripted IL-6. (A) The mRNA and protein levels of IL-6 in subcutaneous tumors were measured by real-time PCR and ELISA, respectively. (B) The phosphorylation of STAT3 reduced by CBS overexpression along with or without IL-6 recombinant protein treatment. (C) Transcription factors array showed that 14 transcription factors were significantly reduced along with CBS overexpression. (D) The 14 transcription factors affected by CBS overexpression was verified by real-time PCR. (E) The overexpression efficiency of JUN, JUNB, NKX2-5 and PRRX2 was determined by real-time PCR. (F) CBS stable overexpression or control MHCC97H cells were transfected with JUN, JUNB, NKX2-5 or PRRX2 plasmid, then the mRNA level of IL-6 was analyzed by real-time PCR. (G and H) CBS stable knockdown or control Hep3B cells were transfected as indicated, then the mRNA level of PRRX2 (G) and IL-6 (H) was analyzed by real-time PCR. (I and J) The correlation between CBS and PRRX2 expression in HCC clinical samples ( $n=236)$ was analyzed by Pearson's r test. In A and $\mathrm{C}-\mathrm{H}$, data are represented as mean $\pm \mathrm{SEM}$, and statistical significance was calculated by Student's two-tailed t-test. ${ }^{*} \mathrm{P}<0.05$; ${ }^{* *} \mathrm{p}<0.01$; ${ }^{* \star *} \mathrm{p}<0.001$. CBS, cystathionine $\beta$-synthase; HCC, hepatocellular carcinoma; IL, interleukin; NS, no significance. 
A

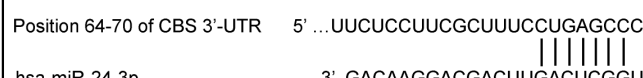

hsa-miR-24-3p

3. GACAAGGACGACUUGACUCGG

B

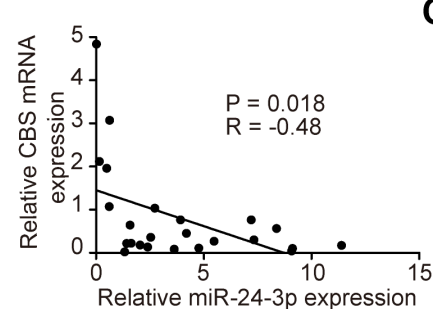

C

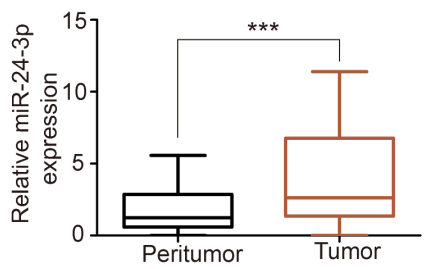

D

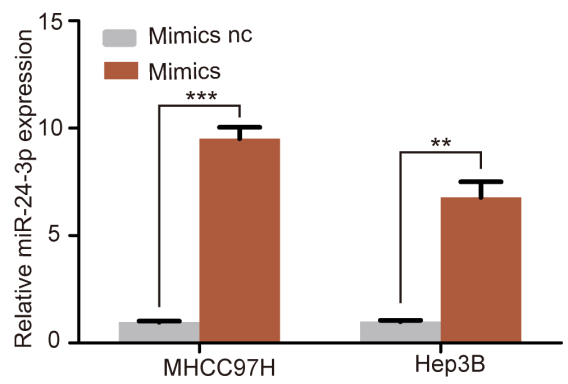

E
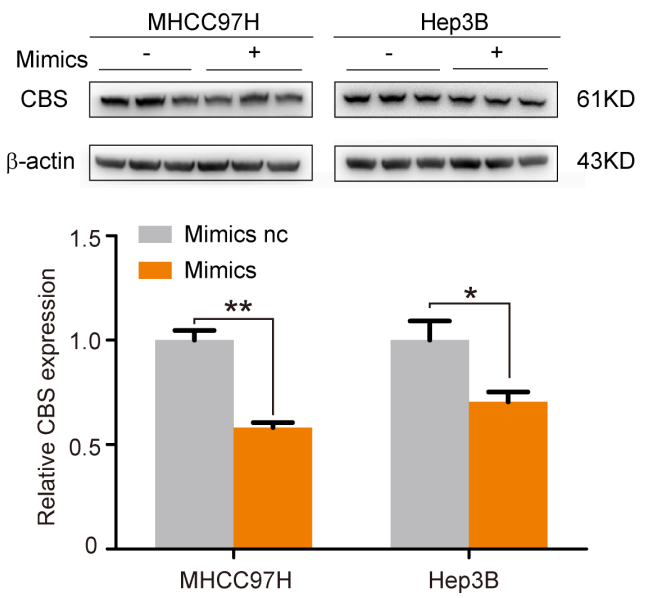

$\mathbf{F}$
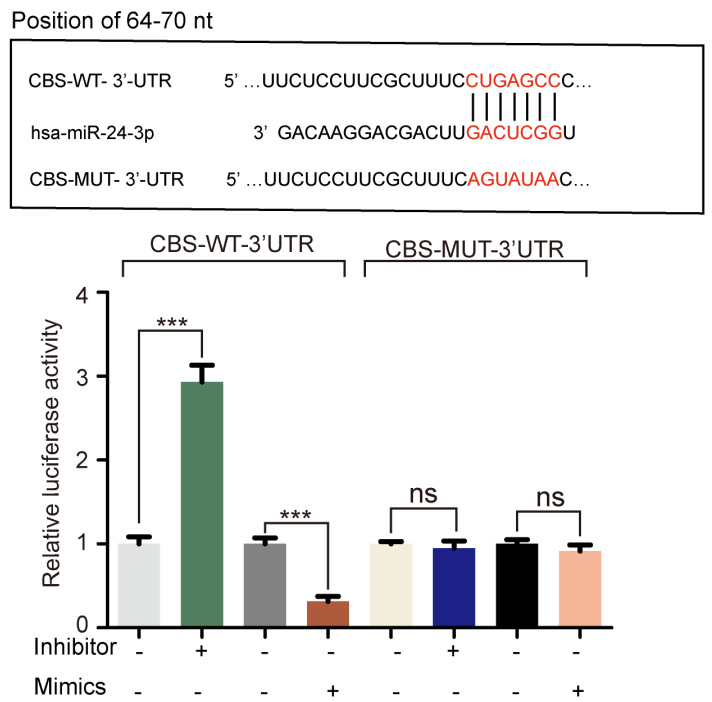

G
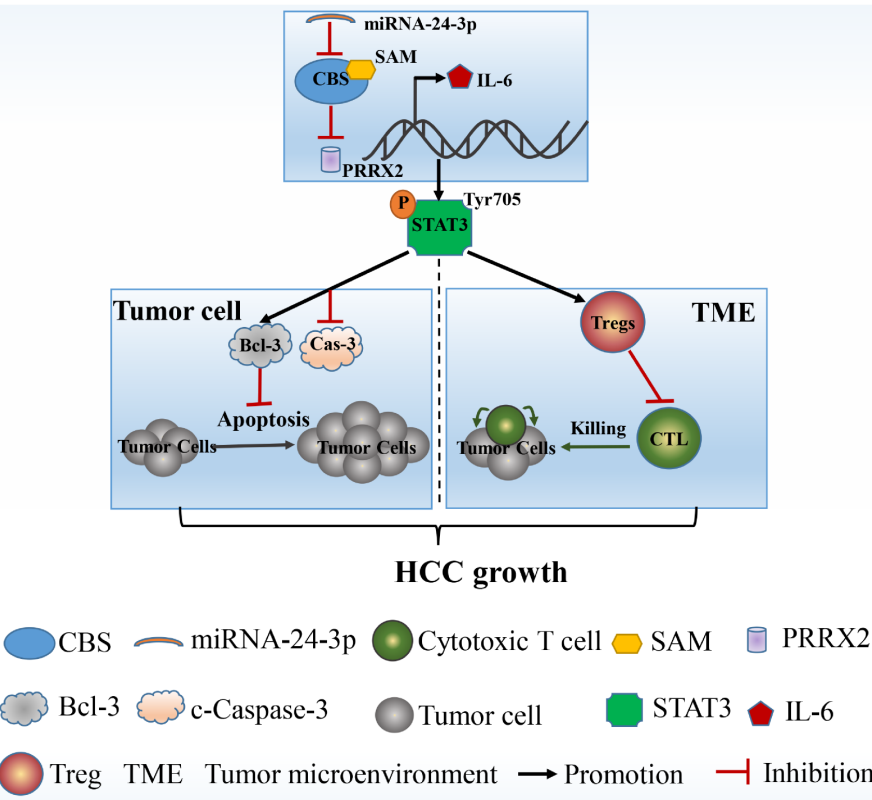

Figure 7 miR-24-3p is an upstream suppressor of CBS. (A) The TargetScan database was used to show that miR-24-3p had sequences complementary to CBS 3'-UTR. (B) The correlation between CBS and miR-24-3p expression in HCC tumor tissues $(n=24)$. (C) The expression level of miR-24-3p in HCC tumor tissues and paired adjacent peritumor specimens were investigated by real-time PCR $(n=24)$. ( $D$ and E) MHCC97H and Hep3B cells were transfected with miR-24-3p or control mimics, then the expression of miR-24-3p was detected by real-time PCR (D), and the protein level of CBS was detected by western blot (E). (F) Complementary sequence between CBS 3'UTR and miR-24-3p, and the effect of miR-24-3p mimics or its inhibitor on the luciferase activity of wild or mutant CBS was tested by luciferase reporter assay. (G) Hypothetical scheme for the mechanisms of CBS inhibiting HCC growth. In B, data are represented as min-to-max bar graphs with median lines; in D-F, data are represented as mean \pm SEM, and statistical significance was calculated by Student's two-tailed t-test $(B, D-F)$. ${ }^{*} P<0.05$; ${ }^{* \star} p<0.01 ;{ }^{* \star *} p<0.001$. CBS, cystathionine $\beta$-synthase; HCC, hepatocellular carcinoma; NS, no significance.

we found that miR-942-5p, miR-139-3p, miR-24-3p and miR-1303 had sequences complementary to CBS 3'-UTR (figure 7A and online supplemental figure 8A), with score values greater than 80 in the TargetScan database. We then examined the expression levels of these four miRNAs in 24 HCC tissues. Further analysis showed that the level of miR-24-3p was inversely associated with CBS mRNA level, whereas the correlation between
CBS mRNA level and the other three miRNAs were not significant (figure $7 \mathrm{~B}$ and online supplemental figure $8 \mathrm{~B})$. Also, the level of miR-24-3p was increased in HCC samples compared with peritumor tissues (figure 7C). To verify the hypothesis that miR-24-3p might be an upstream regulator of CBS, we transfected MHCC97H and Hep3B cells with miR-24-3p mimics, and results showed that miR-24-3p overexpression led to a decrease 
of CBS protein level in both MHCC97H and Hep3B cells (figure 7D,E). Next a luciferase reporter assay was performed to further validate whether miR-24-3p was a direct regulator of CBS. When cotransfected with Wt-CBS 3'-UTR luciferase reporter plasmid, miR-24-3p mimics led to a significant decrease in the luciferase activity of CBS, whereas the luciferase activity of Mut-CBS 3'UTR was not affected by miR-24-3p mimics (figure 7F). In addition, cotransfection with miR-24-3p inhibitor led to a significant increase in the luciferase activity of Wt-CBS 3'-UTR, while this effect was not observed in Hep3B cells transfected with Mut-CBS 3'UTR (figure 7F). Taken together, miR-24-3p directly targeted CBS and negatively regulated the expression of CBS, suggesting that miR-24-3p was an upstream repressor of CBS in HCC.

\section{DISCUSSION}

CBS expression was heterogeneous in diverse cancers. It was shown to be elevated in colorectal, ovarian and thyroid malignancy. ${ }^{28} 38$ However, in the present study, we found that CBS was significantly downregulated in HCC and was associated with poor prognosis in HCC patients (figure 1A-I), a probable reason might be the abundant expression of CBS in liver tissue (online supplemental figure 1). CBS has been demonstrated to promote tumor growth via enhancing glycolysis and angiogenesis in colorectal and ovarian cancers. ${ }^{28}$ However, in our study, CBS exhibited little effect on these two factors in HCC (online supplemental figure 4). Instead, we found that CBS downregulation directly reduced HCC cell apoptosis, while also enhanced tumor-infiltrating of Tregs that inhibited cytotoxic $\mathrm{CD}^{+} \mathrm{T}$ cells, and all these functions were dependent on the activation of IL-6/STAT3 signaling pathway (figure 7G). Furthermore, we identified PRRX2 as a transcription factor of IL-6, and PRRX2 was downregulated by CBS, and miR-24-3p was proven to be an upstream suppressor of CBS in HCC.

So far, the potential role of CBS in regulating tumorous microenvironment in HCC has not yet been reported. Our results demonstrated that Cbs deficiency facilitated tumorous infiltration of Tregs while repressed cytotoxic $\mathrm{T}$ cells infiltrating and its tumor killing capacity (figure $4 \mathrm{I}-4 \mathrm{~N}$ ). In addition, Cbs deficiency-mediated IL-6/STAT3 activation also promoted the expression of Foxp3 in tumor-infiltrating Tregs (figure 5J), which was consistent with previous findings that STAT3 could directly bind to the promoter of Foxp3 and facilitate its transcriptional expression in $\mathrm{CD} 4^{+} \mathrm{CD} 25^{+}$Treg cells. ${ }^{39} 40$ As a regulator for Treg/Th17 balance, IL-6 was shown to induce the development of Th17 cells from naive T cells together with TGF- $\beta$; in contrast, IL-6 inhibited TGF- $\beta$ induced Treg differentiation. ${ }^{41}$ In the present study, we found that Cbs deficiency increased the expression of IL-6 in Hepa1-6-homografted tumor tissues and the abundance of tumor-infiltrating Tregs and the expression of Foxp3 in these infiltrated Tregs in a STAT3-dependent manner (figures 5I,J and 6A). Besides, Cbs deficiency also improved IL-6 and IL-10 levels in tumor-infiltrating Tregs (online supplemental figures 5I and 6C), while TGF- $\beta$ was almost unchanged in CBS-deficient tumor tissues compared with wild type tumor tissues (data not shown). Whether CBS has an influence on Treg differentiation in tumor microenvironment of HCC needs to be further investigated.

$\mathrm{H}_{2} \mathrm{~S}$ has been proven to provide significant hepatoprotection by inhibiting the IL-6/STAT3 axis, ${ }^{42}$ while was also showed to promote HCC cell proliferation by activating the STAT3-COX2 pathway. ${ }^{43}$ Another study investigating the relationship between $\mathrm{H}_{2} \mathrm{~S}$ concentration and inflammation found that lower concentration $(100-200 \mu \mathrm{M})$ of NaHS inhibited LPS-evoked cytokine formation, while higher concentration of NaHS increased the synthesis of cytokines in RAW 264.7 macrophage cell line. ${ }^{44}$ Our previous study also demonstrated that $200 \mu \mathrm{M}$ of NaHS restrained the production of IL- 6 induced by LPS in mouse peritoneal macrophages, ${ }^{18}$ and in this study, we found that CBS was downregulated in HCC, and the basal level of $\mathrm{H}_{2} \mathrm{~S}$ was relatively low in HCC tumor tissues (figure 1A-G and online supplemental figure 2A). We speculated that the priority function of $\mathrm{H}_{2} \mathrm{~S}$ at low concentration was to inhibit certain signaling pathways responsible for cytokine production, while excess $\mathrm{H}_{2} \mathrm{~S}$ might bring acute stress and elicit cytokine release by activating related pathways.

The regulatory function of the $\mathrm{CBS} / \mathrm{H}_{2} \mathrm{~S}$ axis on IL-6 expression has been widely reported, but the specific mechanism remains unclear. While transcription factors JUN and JUNB have been showed to directly regulate IL-6 transcription, ${ }^{45} 46$ we identified two more transcription factors NKX2-5 and PRRX2 by using a transcription factors array that could also promote IL-6 expression. However, only PRRX2 was proven to be a mediator of CBS downregulated IL-6 production in HCC cells (figure 6C-H).

According to bioinformatics prediction algorithms, miR-24-3p has a complementary binding sequence to the 3'-UTR of CBS. miR-24-3p has also been revealed to be upregulated in several cancers including $\mathrm{HCC}^{47}$ which is consistent with our current findings (figure 7C). We also demonstrated that miR-24-3p expression level was negatively correlated to CBS expression in HCC clinical samples (figure 7B), and further confirmed miR-24-3p was an upstream suppressor of CBS in HCC cells by using its mimics and inhibitor (figure 7D-F). Besides, we detected the expression of miR-24-3p in colorectal and ovarian tumors and found that miR-24-3p expression level was remarkably lower in tumor tissues compared with matched pericarcinomatous tissues, which was consistent with a previous research, ${ }^{48}$ but miR-24-3p expression still exhibited negative correlations with CBS mRNA level in colorectal and ovarian tumor tissues (online supplemental figure 9). Therefore, miR-24-3p expression might be another reason why CBS behaves differently between HCC and other tumors.

In summary, our findings highlighted critical roles of CBS in regulating HCC that had not been explored 
before, especially in remodeling tumor microenvironment. Detailed, we demonstrated that downregulation of CBS in HCC inhibited tumor cell apoptosis and accelerated tumor infiltration of Tregs, while impeded cytotoxic T cells infiltrating and its tumor killing capacity, and these effects were all blocked by IL-6/STAT3 inactivation. These data suggest CBS as a new potential target for HCC treatment, especially in immunotherapy.

\section{Author affiliations}

${ }^{1}$ Department of Liver Surgery, Liver Cancer Institute, Zhongshan Hospital, Fudan University \& Key Laboratory of Carcinogenesis and Cancer Invasion of Ministry of Education \& Research Unit of Liver cancer Recurrence and Metastasis, Chinese Academy of Medical Sciences, Shanghai, People's Republic of China ${ }^{2}$ Department of Immunology and Pathogenic Biology, School of Basic Medical Sciences, Shanghai University of Traditional Chinese Medicine, Shanghai, People's Republic of China

${ }^{3}$ Department of Biochemistry and Molecular, School of Basic Medical Sciences, Fudan University, Shanghai, People's Republic of China

${ }^{4}$ Institutes of Biomedical Sciences, Fudan University, Shanghai, People's Republic of China

Contributors Y-FZ, S-SS, M-XT and ZT: experimental design; acquisition of data; analysis and interpretation of data; drafting of the manuscript; and statistical analysis; HW, YF, W-FQ, X-FJ and C-YT: acquisition of data and critical revision of the manuscript; RH, P-YZ, S-GZ, JZ and JF: material support and critical revision of the manuscript; Y-HS and W-RL: study concept and design; study supervision; and critical revision of the manuscript for important intellectual content.

Funding This work was supported by the grants from National Natural Science Foundation of China $(82073217,81800790,81902963,81773067,82073218$ and 82003084), Shanghai Municipal Science and Technology Major Project (2018SHZDZX05), Shanghai Municipal Key Clinical Specialty, CAMS Innovation Fund for Medical Sciences (2019-I2M-5-058), National Key R\&D Program of China (2020YFE0202200), China Postdoctoral Science Foundation (2018M640343, 2019T120305) and Shanghai Sailing Program (19YF1407800).

Competing interests No, there are no competing interests.

\section{Patient consent for publication Not required}

Ethics approval This research has been approved by the Clinical Research Ethics Committee of the Shanghai Zhongshan Hospital (Fudan University, Y2019-033). All patients were informed of the aim of the study and gave consent to donate their samples. All animal care and experimental protocols were approved by the Animal Ethics Committees of Zhongshan Hospital (Fudan University) and carried out according to the Animal Management Rules of the Ministry of Health of China.

Provenance and peer review Not commissioned; externally peer reviewed.

Data availability statement Data are available on reasonable request. All data relevant to the study are included in the article or uploaded as supplementary information. The datasets generated during and/or analyzed during the current study are available from the corresponding author on reasonable request.

Supplemental material This content has been supplied by the author(s). It has not been vetted by BMJ Publishing Group Limited (BMJ) and may not have been peer-reviewed. Any opinions or recommendations discussed are solely those of the author(s) and are not endorsed by BMJ. BMJ disclaims all liability and responsibility arising from any reliance placed on the content. Where the content includes any translated material, BMJ does not warrant the accuracy and reliability of the translations (including but not limited to local regulations, clinical guidelines, terminology, drug names and drug dosages), and is not responsible for any error and/or omissions arising from translation and adaptation or otherwise.

Open access This is an open access article distributed in accordance with the Creative Commons Attribution Non Commercial (CC BY-NC 4.0) license, which permits others to distribute, remix, adapt, build upon this work non-commercially, and license their derivative works on different terms, provided the original work is properly cited, appropriate credit is given, any changes made indicated, and the use is non-commercial. See http://creativecommons.org/licenses/by-nc/4.0/.

\section{ORCID iD}

Ying-Hong Shi http://orcid.org/0000-0002-1833-8988
REFERENCES

1 Sung H, Ferlay J, Siegel RL, et al. Global cancer statistics 2020: GLOBOCAN estimates of incidence and mortality worldwide for 36 cancers in 185 countries. CA Cancer J Clin 2021;71:209-49.

2 Llovet JM, Zucman-Rossi J, Pikarsky E, et al. Hepatocellular carcinoma. Nat Rev Dis Primers 2016;2:16018.

3 Jörs S, Jeliazkova P, Ringelhan M, et al. Lineage fate of ductular reactions in liver injury and carcinogenesis. $J$ Clin Invest 2015;125:2445-57.

4 Grivennikov SI, Greten FR, Karin M. Immunity, inflammation, and cancer. Cell 2010;140:883-99.

5 Hernandez-Gea V, Toffanin S, Friedman SL, et al. Role of the microenvironment in the pathogenesis and treatment of hepatocellular carcinoma. Gastroenterology 2013;144:512-27.

6 Ringelhan M, Pfister D, O'Connor T, et al. The immunology of hepatocellular carcinoma. Nat Immunol 2018;19:222-32.

7 Quail DF, Joyce JA. Microenvironmental regulation of tumor progression and metastasis. Nat Med 2013;19:1423-37.

8 Tavazoie MF, Pollack I, Tanqueco R, et al. LXR/ApoE activation restricts innate immune suppression in cancer. Cell 2018;172:825-40.

9 Cassetta L, Pollard JW. Targeting macrophages: therapeutic approaches in cancer. Nat Rev Drug Discov 2018;17:887-904.

10 Shipp C, Speigl L, Janssen N, et al. A clinical and biological perspective of human myeloid-derived suppressor cells in cancer. Cell Mol Life Sci 2016;73:4043-61.

11 Fu J, Zhang Z, Zhou L, et al. Impairment of CD4+ cytotoxic T cells predicts poor survival and high recurrence rates in patients with hepatocellular carcinoma. Hepatology 2013;58:139-49.

12 Plitas G, Rudensky AY. Regulatory T cells: differentiation and function. Cancer Immunol Res 2016;4:721-5.

13 Noy R, Pollard JW. Tumor-Associated macrophages: from mechanisms to therapy. Immunity 2014;41:49-61.

14 Gabrilovich DI, Nagaraj S. Myeloid-Derived suppressor cells as regulators of the immune system. Nat Rev Immunol 2009;9:162-74.

15 Braunstein AE, Goryachenkova EV, Lac ND. Reactions catalysed by serine sulfhydrase from chicken liver. Biochim Biophys Acta 1969;171:366-8.

16 Kimura $\mathrm{H}$. Signaling molecules: hydrogen sulfide and polysulfide. Antioxid Redox Signal 2015;22:362-76.

17 Zhou Y-F, Wu X-M, Zhou G, et al. Cystathionine $\beta$-synthase is required for body iron homeostasis. Hepatology 2018;67:21-35.

18 Zhang M-W, Yang G, Zhou Y-F, et al. Regulating ferroportin-1 and transferrin receptor-1 expression: a novel function of hydrogen sulfide. J Cell Physiol 2019;234:3158-69.

19 Zhong Z, Wen Z, Darnell JE. Stat3: a STAT family member activated by tyrosine phosphorylation in response to epidermal growth factor and interleukin-6. Science 1994;264:95-8.

20 Naugler WE, Sakurai T, Kim S, et al. Gender disparity in liver cancer due to sex differences in MyD88-dependent IL-6 production. Science 2007;317:121-4.

21 Gerdes J, Schwab U, Lemke H, et al. Production of a mouse monoclonal antibody reactive with a human nuclear antigen associated with cell proliferation. Int J Cancer 1983;31:13-20.

22 Mao C, Liu X, Zhang Y, et al. DHODH-mediated ferroptosis defence is a targetable vulnerability in cancer. Nature 2021;593:586-90.

23 Crowley LC, Waterhouse NJ. Detecting cleaved caspase-3 in apoptotic cells by flow cytometry. Cold Spring Harb Protoc 2016;2016. doi:10.1101/pdb.prot087312. [Epub ahead of print: 0111 2016].

24 Camp RL, Dolled-Filhart M, Rimm DL. X-tile: a new bio-informatics tool for biomarker assessment and outcome-based cut-point optimization. Clin Cancer Res 2004;10:7252-9.

$25 \mathrm{Kim} \mathrm{J}$, Hong SJ, Park JH, et al. Expression of cystathionine beta-synthase is downregulated in hepatocellular carcinoma and associated with poor prognosis. Oncol Rep 2009;21:1449-54

26 Chao C, Zatarain JR, Ding Y, et al. Cystathionine-Beta-Synthase inhibition for colon cancer: enhancement of the efficacy of aminooxyacetic acid via the prodrug approach. Mol Med 2016;22:361-79.

27 Majtan T, Pey AL, Gimenez-Mascarell P, et al. Potential pharmacological chaperones for cystathionine beta-synthasedeficient homocystinuria. Handb Exp Pharmacol 2018;245:345-83.

28 Hellmich MR, Coletta C, Chao C, et al. The therapeutic potential of cystathionine $\beta$-synthetase/hydrogen sulfide inhibition in cancer. Antioxid Redox Signal 2015;22:424-48.

29 Vaughan RA, Gannon NP, Garcia-Smith R, et al. $\beta$-alanine suppresses malignant breast epithelial cell aggressiveness through alterations in metabolism and cellular acidity in vitro. Mol Cancer 2014;13:14.

30 Poznanski SM, Singh K, Ritchie TM, et al. Metabolic flexibility determines human NK cell functional fate in the tumor microenvironment. Cell Metab 2021;33:1205-20. 
31 Zhou Z, Christofidou-Solomidou M, Garlanda C, et al. Antibody against murine PECAM-1 inhibits tumor angiogenesis in mice. Angiogenesis 1999;3:181-8.

32 Zhou X, Cao Y, Ao G, et al. CaMKK $\beta$-dependent activation of AMP-activated protein kinase is critical to suppressive effects of hydrogen sulfide on neuroinflammation. Antioxid Redox Signal 2014;21:1741-58.

$33 \mathrm{Kim}$ H-P, Leonard WJ. CREB/ATF-dependent T cell receptor-induced FOXP3 gene expression: a role for DNA methylation. J Exp Med 2007;204:1543-51.

34 Kohli RM, Zhang Y, enzymes TET. Tet enzymes, TDG and the dynamics of DNA demethylation. Nature 2013;502:472-9.

35 Yang R, Qu C, Zhou Y, et al. Hydrogen sulfide promotes Tet1and Tet2-Mediated FOXP3 demethylation to drive regulatory $T$ cell differentiation and maintain immune homeostasis. Immunity 2015;43:251-63.

36 Wang $\mathrm{P}$, Isaak CK, Siow YL, et al. Downregulation of cystathionine $\beta$-synthase and cystathionine $\gamma$-lyase expression stimulates inflammation in kidney ischemia-reperfusion injury. Physiol Rep 2014;2:e12251.

37 Hirano T, Ishihara K, Hibi M. Roles of STAT3 in mediating the cell growth, differentiation and survival signals relayed through the IL-6 family of cytokine receptors. Oncogene 2000;19:2548-56.

38 Turbat-Herrera EA, Kilpatrick MJ, Chen J, et al. Cystathione $\beta$-synthase is increased in thyroid malignancies. Anticancer Res 2018;38:6085-90.

39 Kortylewski M, Xin H, Kujawski M, et al. Regulation of the IL-23 and IL-12 balance by STAT3 signaling in the tumor microenvironment. Cancer Cell 2009;15:114-23.
40 Wang $Y$, Shen $Y$, Wang S, et al. The role of STAT3 in leading the crosstalk between human cancers and the immune system. Cancer Lett 2018;415:117-28.

41 Kimura A, Kishimoto T. II-6: regulator of Treg/Th17 balance. Eur J Immunol 2010;40:1830-5.

42 Fouad AA, Hafez HM, Hamouda A. Hydrogen sulfide modulates IL-6/STAT3 pathway and inhibits oxidative stress, inflammation, and apoptosis in rat model of methotrexate hepatotoxicity. Hum Exp Toxicol 2020;39:77-85.

43 Zhen Y, Wu Q, Ding Y, et al. Exogenous hydrogen sulfide promotes hepatocellular carcinoma cell growth by activating the STAT3-COX-2 signaling pathway. Oncol Lett 2018;15:6562-70.

44 Whiteman M, Li L, Rose P, et al. The effect of hydrogen sulfide donors on lipopolysaccharide-induced formation of inflammatory mediators in macrophages. Antioxid Redox Signal 2010;12:1147-54.

45 Pflegerl P, Vesely P, Hantusch B, et al. Epidermal loss of JunB leads to a SLE phenotype due to hyper IL-6 signaling. Proc Natl Acad Sci U S A 2009;106:20423-8.

46 Krause A, Holtmann H, Eickemeier S, et al. Stress-activated protein kinase/Jun $\mathrm{N}$-terminal kinase is required for interleukin (IL)-1-induced IL-6 and IL-8 gene expression in the human epidermal carcinoma cell line KB. J Biol Chem 1998;273:23681-9.

47 Dong X, Ding W, Ye J, et al. MiR-24-3p enhances cell growth in hepatocellular carcinoma by targeting metallothionein $1 \mathrm{M}$. Cell Biochem Funct 2016;34:491-6.

48 Gao Y, Liu Y, Du L, et al. Down-Regulation of miR-24-3p in colorectal cancer is associated with malignant behavior. Med Oncol 2015;32:362. 